\title{
THE DRIVER HAS CONTROL: EXPLORING DRIVING PERFORMANCE WITH VARYING AUTOMATION CAPABILITIES
}

\author{
Mishel Johns, David B Miller, Annabel C Sun, Shawnee Baughman, Tongda Zhang, Wendy Ju \\ Stanford University \\ Stanford, California, USA \\ mishel@stanford.edu
}

\begin{abstract}
Summary: As vehicle automation becomes more capable and prevalent, an understanding of how drivers will interact with automation systems of varying capabilities will be of critical importance. In this study, we compare the performance of drivers on takeover of control from varying types of automation systems (single-function and combined function). Participants drove a 20-minute course with sections of automated driving, and with several traffic events designed to elicit a driver response. Structured transfers of control between automated and manual driving modes occurred following a 7-second countdown at fixed locations on the course. Significant differences were found between groups in terms of lanekeeping ability immediately after taking control following a period of automated vehicle control or partial driver/automation control, but significant differences were not found in accident evasion ability, even five seconds after resuming full control.
\end{abstract}

\section{INTRODUCTION}

This study explores whether driver reactions are impaired immediately after a structured transition from automated driving to manual driving. This understanding is crucial to the design of automated vehicles up to NHTSA Level 3 (L3), where human intervention will be necessary when the computer controlling the vehicle is unable to handle the challenges presented. We consider three hypotheses with implications for automated driving safety and thus for the design of automated systems to work with human constraints: The attention resource degradation hypothesis would suggest that as the driver is not actively focused on driving while the vehicle is in automated mode and attention resources may shrink to accommodate reduction in demand (Young \& Stanton, 2002), when the attention demand rises very quickly upon transfer of control to the driver, performance may be inadequate to ensure safe driving. Billings' (Billings, 1991) and Weiner's (Wiener, 1989) papers discuss how automation of aircraft systems reduces cognitive workload in low-load conditions such as cruise flight, but may increase workload in situations where workload is already high, such as during climbs and descents. In aircraft, this effect increases with the degree of automation. In the context of automated road vehicles, this translates to a lower workload when supervising the automated system during routine highway driving, but may result in a higher workload and reduced performance during more complex situations where the system might fail or require human input.

The attention resource conservation hypothesis, which follows from the limited capacity theories noted by Kahneman (Kahneman, 1973) and Wickens (Wickens, 1980), and the depletion and replenishment research of Ariga and Lleras (Ariga \& Lleras, 2011), would suggest that as attention demand is reduced during automated driving, the driver can rest and thus replenish their 'reservoir' of cognitive resources. When cognitive resources are demanded, such as when facing a potential accident, resources can then be deployed to respond to address the situation at hand. 
The compensation hypothesis states that the driver recognizes and compensates for higher workload demand. Sanders and Baron (Sanders \& Baron, 1975) found that simple tasks can actually increase performance in the primary task due to a compensatory process, possibly an increase in the general motivation level of the participant. For more complex tasks, however, such distractions reduce performance. Prior research in the domain of automated driving has not yet yielded definitive answers regarding the issue of cognitive resource allocation: Stanton et.al. (N. A. Stanton, Young, \& McCaulder, 1997) posit that Adaptive Cruise Control (ACC) can reduce the driver's cognitive workload and might leave the driver underloaded, but did not find significant performance differences between drivers in the manual driving and the ACC condition. It may be that attention resources are throttled back in cases of underload, in response to reduced demand (Young \& Stanton, 2002). Conversely, Merat and Jamson (Merat \& Jamson, 2008) state that when drivers had to supervise driving in a highly automated vehicle, they performed worse than normal on responding to critical events where they had to take over control of their own accord, leaving this an open question for investigation.

To investigate this issue, we compared the driving performance of participants using different types of automated driving systems - Automated Steering Control (AS), ACC, AS+ACC - with those who drove without assistance. In our simulated driving course, critical events (pedestrian incursions, cars cutting off the driver, and a leading car suddenly slowing) were arranged to occur, shortly after transition to manual driving in the automated conditions. In the automated conditions, the vehicle controlled either the steering, the speed, or both during portions of the drive when it would make sense to allow the automated driving system to control the car in an L3 driving scenario. This study built upon previous research by Lee, Joo, \& Nass (2014), studying switched-state vehicle automation under the same automation conditions.

\section{METHOD}

\section{Conditions: Automation Functionality}

The participants operated the simulated vehicle in manual mode and while in certain sections, in one of these four conditions (between-subjects variable):

- Manual Driving $(n=12)$ : The driver had unassisted manual control for the entire course.

- Automated Steering $(A S)(n=10)$ : During the automated sections, the driver controlled the brakes and throttle, while the automated system controlled the steering. The steering wheel in the car was decoupled while the computer controlled vehicle steering, with the road torque being reapplied to the steering wheel when control was passed back to the driver. The automated steering system did not swerve around obstacles.

- Adaptive Cruise Control (ACC) $(n=10)$ : The participant controlled the steering, while the automated system controlled the brake and the throttle during the automated driving sections. The system automatically braked and accelerated to maintain speed and headway, and would slow or stop in response to dangers in the road like a car cutting in ahead.

- Fully Automated mode $(n=14)$ : AS and ACC are combined, and the system kept the car in the lane, maintained speed and headway, and responded to dangers by braking.

In all of these conditions, the automation could not be disabled by the driver; while this is unlike the design of an actual automated systems, it allowed us to more carefully control the study. 


\section{Course Design}

The experiment was conducted at the Stanford Driving Simulator, a full-car high-fidelity simulator with a 270-degree field of view screen and a full vehicle chassis. A vehicle dynamics model applied simulated road torque to the steering wheel. The participants spent approximately 20 minutes driving a $26.5 \mathrm{~km}$ course, which included road sections with speed limits of 75, 35, and 25 miles per hour (four lane divided, four lane undivided, and two lane undivided roads, respectively). They were not allowed to use personal devices or engage in other distracting activities during the study. They drove through five sections of automated driving interspersed with manual driving sections (see Figure 1). Participants were not explicitly given reasons for the transfers of control. Over the course of the study, the car encountered five "events" in the course: two pedestrian incursions into the road, two instances of a car cutting into the participant's lane, and one of a car stopping without warning in front of the participant. Two of the events occurred in the automated driving sections, and only the participants in the automated steering or manual driving conditions had to respond to them, as in the fully automated or ACC conditions, the car would automatically brake to avoid the hazard. The other three occurred in manual mode for all participants. At the speed limit, these three events occur 5, 10 and 15 seconds after the transfer of control. The events and the structured transfer of control were triggered at specific locations on the course.

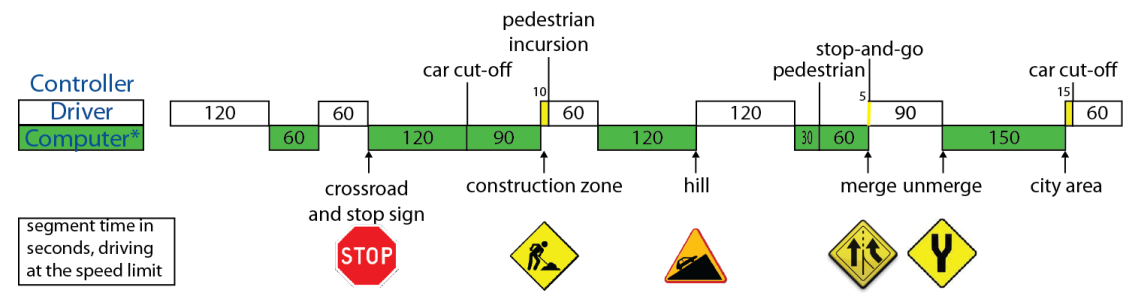

Figure 1. Outline of experiment course.

Times are denoted in seconds; for the manual components, times are target times at the zone speed limit

All transfers of control and critical events occurred on straight road segments, which limited the difficulty of evading the potential accidents. This was done to reduce driver discomfort driving on curved roads, and to make it possible to measure driver lane-keeping performance.

\section{Transfer of control}

The transfer of control from fully or partially automated driving modes to driver control occurred at certain locations on the course, over a seven second window (see Fig 2). After a two-secondlong audio message asking participants to "Please disable automation," there was a five second visual countdown on the instrument cluster, after which the car transfers control. The participants 
were asked to press a button on the steering wheel (see Fig 3) to acknowledge that they were ready to take over control from the vehicle during the five-second transition window.

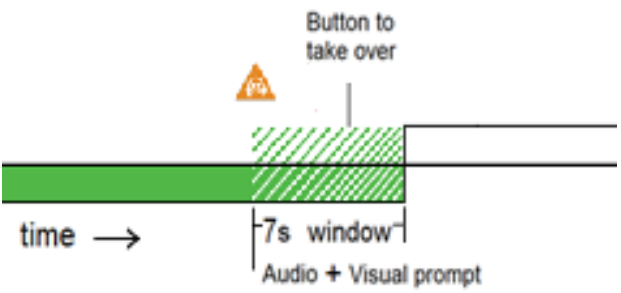

Figure 2. Automated control to manual control transition window

Pressing the button would change the color of the automation icon on the instrument panel (see Fig 4). The transfer of control always occurred at the end of this structured transition window, regardless of when or whether the button was pressed, but participants were not informed of this.
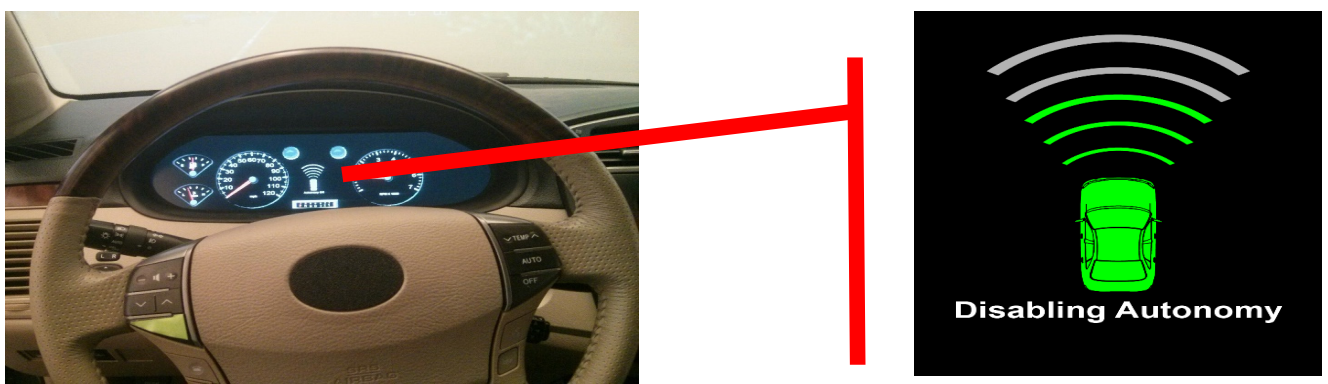

Figure 3. Steering wheel with mode switch button marked in green. The same button enables or disables the automated driving features, and the mode switch is only enabled in specific transition zones
Figure 4. Automation system status indicator on instrument panel. The car icon lights up in green to indicate that the system is on, and turns to grey to when it is off. The arcs in front of the car show when the system is turning on or off, and descriptive text is provided below the vehicle icon

\section{Participants}

Forty-seven (47) undergraduate and graduate students at Stanford University between the ages of 18 and 24 (M: 21.5 years old, S.D: 3.4) participated in this study. All possessed a valid driver's license, and a minimum of two years of experience driving (M: 4.8, SD: 2.8) on the right-side of the road. Participants were remunerated with either a gift card or course credit.

\section{RESULTS AND DISCUSSION}

Simulator data, including vehicle state, position, velocity and driver inputs such as brake, steering and throttle, was captured at $60 \mathrm{~Hz}$. Data on speed limit violations, lane position, and collision with other vehicles were also collected. Important time points, like the moment of transfer of control from the car to the driver, were marked in the data for analysis.

\section{Driving performance on transfer of control from the automated system to the driver}


Steering movements post transition: The standard deviation of the steering angle (Vries \& Waard, 1991) was calculated over a four second period after transfer of control beginning at transition time, and averaged for each participant across four transfers. The four second duration was chosen because it closes before the start of the critical events placed after transfer of control, the closest of which was at five seconds. A one-way ANOVA showed significant differences between the groups $[\mathrm{F}(3,43)=4.109, p<.05]$. Post-hoc comparison using the Tukey HSD indicated that the AS $(\mathrm{M}=0.06, \mathrm{SD}=0.06)$ and the manual $(\mathrm{M}=0.01, \mathrm{SD}=0.01)$ conditions were significantly different from each other $[p<.01]$; the other pairwise tests did not show significant differences (See Fig 5).

However, the large variance in the AS and fully automated conditions may also be a consequence of the implementation of steering control in the simulator system - the sudden application of road torque to the previously decoupled steering wheel on transfer of control may have influenced the lane-keeping actions of drivers. While participants were asked to not turn the steering wheel when the vehicle was in control of steering, it is likely that participants who were in partial control (AS condition) were more likely to attempt to turn it, causing a jerk in the road wheel angle when control was returned to the driver. This hypothesis is supported by the correlation $[\mathrm{r}(45)=0.90, p<.01]$ between the deviation of the steering wheel from the center position at the moment of takeover and the standard deviation of the steering movement in the four-second period after it.

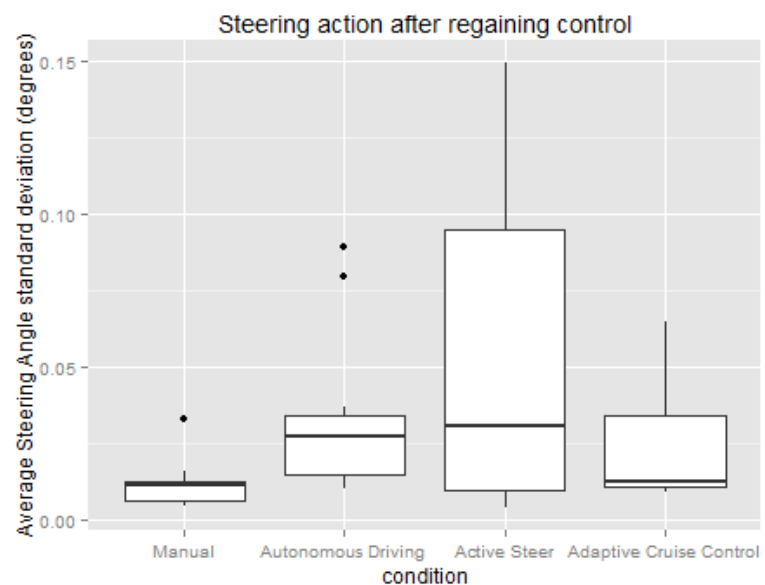

Figure 5. Steering performance after transfer of control measured by std. dev. of steering wheel angle in radians (For data from the manual condition, it is the performance for the same duration of time from roughly the same start position)

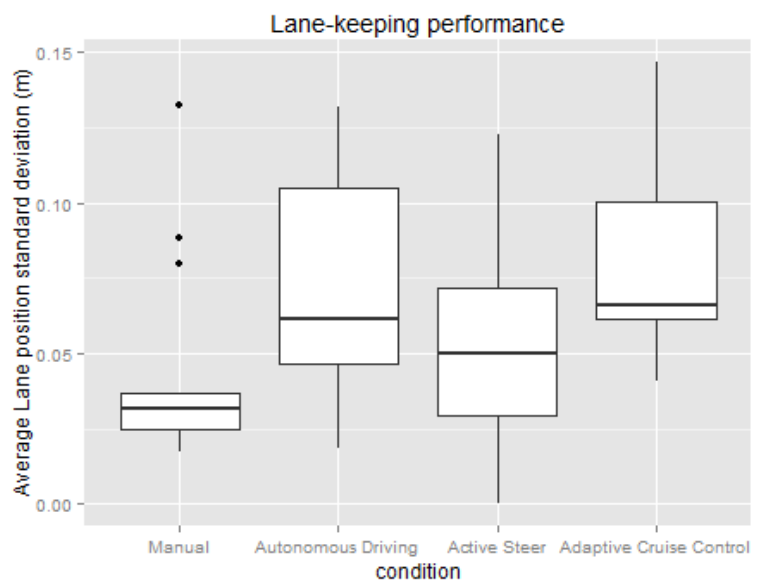

Figure 6. Lane-keeping performance measured by std. dev. of position from center of lane. [Box from the $25^{\text {th }}$ to $7^{\text {th }}$ percentile, bold line shows mean, whiskers extend to furthest point within 1.5 *interquartile range from the box, data points beyond that (outliers) are marked with a dot]

Lane-keeping: We used the standard deviation of the lane position of the car for a period of four seconds immediately after takeover of control as a measure of the driver's control over the vehicle. (Verster \& Roth, 2011; Vries \& Waard, 1991) The averages for each participant over four transfers from automated control to manual control were compared. For participants in the manual driving condition, a four second driving duration starting at approximately the same point was selected. Data from participants who performed a lane change maneuver in the foursecond duration was discarded. Differences between groups were not significant to the 0.05 level $[\mathrm{p}=0.0645]$ (See Fig 6.) 
Delay in pressing the control transfer acknowledgement button: The time from the start of the takeover audio prompt to the pressing of the acknowledge button on the steering wheel was recorded for each participant. We analyzed the mean acknowledgement delay over the five transfers of control to manual driving for each participant. Data from participants that did not press the button within the seven second window was disregarded. There were no statistically significant differences between the groups. A chi-squared test of whether the button was pressed within seven seconds also did not show significant differences between groups.

\section{Performance during events after transfer of control:}

Response time: The duration between the event initiation to the first observed reaction (releasing the throttle, pressing the brakes, or making a large steering input) in the driving data was measured. Statistical analysis showed no significant differences between the conditions. This could be because the duration of time between the transfer of control and the events $(5,10$ and $15 \mathrm{~s}$ ) was too long for the effect of lowered situation awareness during the prior automated/partly automated driving segment to carry over. Since the events are triggered based on location on the course rather than time, moving at higher or lower speeds will change the time to collision for the pedestrian and the cutoff car. This can mean that the perception of danger can be different at different speeds, and this might affect the reaction times.

Success in avoiding the event: There were no statistically significant differences between the conditions in the minimum headway distance to the cutoff car, a measure of success in evasion.

\section{Performance in events while in partial control:}

There were no significant differences between participants in the full manual and the AS conditions who had to take action to respond to events while in partial control.

\section{CONCLUSIONS AND FUTURE WORK}

The results show a significant difference in steering wheel movements within four seconds after takeover of control, with our present implementation of steering wheel torque control.

With a seven second warning for takeover, effects of being in automated modes in the vehicle do not seem to show a significant effect on performance on avoiding critical events five seconds or longer after transfer of control. This could be either due to the events not being challenging enough, or because the total duration of 12 seconds from first cue to the event is sufficient to reduce the effects of the preceding automated section. The variability in the driving performance measures do point towards the compensation hypothesis, in that the participants might be compensating for higher attentional loads during transfer of control, and having a corresponding reduction in the effect of automation on driving performance. It is likely that we are hitting a ceiling effect due to the structured transfer of control and the event not being challenging enough to our participants to show differential effects between conditions. As the complexity of the task increases, however, people's ability to compensate starts to run out, and we could perhaps start to see the effects of attention resource degradation or conservation. The next steps in this research would thus include increasing the challenge of the tasks, and performing the experiment with participants who find driving more challenging: very young or old drivers. Reducing the warning 
time before transfer of control would also help to learn more about handovers of control in automated driving.

\section{ACKNOWLEDGMENTS}

Funding for this study was provided by the Toyota CSRC. This research was started under Prof. Clifford Nass, to whom we owe a debt of gratitude. We would also like to thank our colleagues Brian Mok and David Sirkin for their help in conducting this study.

\section{REFERENCES}

Ariga, A., \& Lleras, A. (2011). Brief and rare mental "breaks" keep you focused: deactivation and reactivation of task goals preempt vigilance decrements. Cognition, 118(3), 439-43.

Billings, C. E. (1991). Human-Centered Aircraft Automation : A Concept and Guideline.

Kahneman, D. (1973). Attention and effort. Englewood Cliffs, NJ: Prentice-Hall.

Lee, K. J., Joo, Y. K., \& Nass, C. (2014). Partially intelligent automobiles and driving experience at the moment of system transition. Proceedings of the 32nd Annual ACM Conference on Human Factors in Computing Systems - CHI '14, 3631-3634.

Merat, N., \& Jamson, A. H. (2008). How do drivers behave in a highly automated car?, 514-521.

National Highway Traffic Safety Administration Preliminary Statement of Policy Concerning Automated Vehicles. (2013).

Sanders, G. S., \& Baron, R. S. (1975). The motivating effects of distraction on task performance. Journal of Personality and Social Psychology, 32(6), 956-963.

Stanton, N. A., Young, M., \& McCaulder, B. (1997). Drive-by-wire: The case of driver workload and reclaiming control with adaptive cruise control. Safety Science, 27(2-3), 149-159.

Stanton, N. A., Young, M. S., Walker, G. H., Turner, H., \& Randle, S. (2001). Automating the Driver's Control Tasks. International Journal of Cognitive Ergonomics, 5(3), 221-236.

Verster, J. C., \& Roth, T. (2011). Standard operation procedures for conducting the on-the-road driving test, and measurement of the standard deviation of lateral position (SDLP). International Journal of General Medicine, 4, 359-71.

Vries, G. D. E., \& Waard, D. D. E. (1991). THE EFFECTS OF MOBILE TELEPHONING ON, 23(4), 309-316.

Wickens, Christopher D. "The structure of attentional resources." Attention and performance VIII 8 (1980).

Wiener, E. L. (1989). Human Factors of Advanced Technology ("Glass Cockpit”) Transport Aircraft, (June).

Young, M. S., \& Stanton, N. a. (2002). Malleable Attentional Resources Theory: A New Explanation for the Effects of Mental Underload on Performance. Human Factors: The Journal of the Human Factors and Ergonomics Society, 44(3), 365-375. 